\title{
Economic evaluation of an extended nutritional intervention in older Australian hospitalized patients: a randomized controlled trial
}

Yogesh Sharma ${ }^{1,2^{*}}$, Campbell Thompson ${ }^{3}$, Michelle Miller ${ }^{4}$, Rashmi Shahi ${ }^{5}$, Paul Hakendorf ${ }^{6}$, Chris Horwood ${ }^{6}$ and Billingsley Kaambwa ${ }^{7}$

\begin{abstract}
Background: Prevalence of malnutrition in older hospitalized patients is 30\%. Malnutrition is associated with poor clinical outcomes in terms of high morbidity and mortality and is costly for hospitals. Extended nutrition interventions improve clinical outcomes but limited studies have investigated whether these interventions are cost-effective.

Methods: In this randomized controlled trial, 148 malnourished general medical patients $\geq 60$ years were recruited and randomized to receive either an extended nutritional intervention or usual care. Nutrition intervention was individualized and started with $24 \mathrm{~h}$ of admission and was continued for 3 months post-discharge with a monthly telephone call whereas control patients received usual care. Nutrition status was confirmed by Patient generated subjective global assessment (PG-SGA) and health-related quality of life (HRQoL) was measured using EuroQoL 5D (EQ-5D-5 L) questionnaire at admission and at 3-months follow-up. A cost-effectiveness analysis was conducted for the primary outcome (incremental costs per unit improvement in PG-SGA) while a cost-utility analysis (CUA) was undertaken for the secondary outcome (incremental costs per quality adjusted life year (QALY) gained).
\end{abstract}

Results: Nutrition status and HRQoL improved in intervention patients. Mean per included patient Australian Medicare costs were lower in intervention group compared to control arm (by \$907) but these differences were not statistically significant (95\% Cl: $-\$ 2956$ to \$4854). The main drivers of higher costs in the control group were higher inpatient $(\$ 13,882$ versus $\$ 13,134)$ and drug ( $\$ 838$ versus $\$ 601)$ costs. After adjusting outcomes for baseline differences and repeated measures, the intervention was more effective than the control with patients in this arm reporting QALYS gained that were higher by 0.0050 QALYs gained per patient ( $95 \%$ Cl: -0.0079 to 0.0199 ). The probability of the intervention being cost-effective at willingness to pay values as low as $\$ 1000$ per unit improvement in PG-SGA was $>98 \%$ while it was $78 \%$ at a willingness to pay $\$ 50,000$ per QALY gained.

Conclusion: This health economic analysis suggests that the use of extended nutritional intervention in older general medical patients is likely to be cost-effective in the Australian health care setting in terms of both primary and secondary outcomes.

Trial registration: ACTRN No. 12614000833662. Registered 6 August 2014.

Keywords: Malnutrition, Economic evaluation, Health related quality of life, Quality adjusted life years, Older patients

\footnotetext{
* Correspondence: Yogesh.Sharma@sa.gov.au

'Department of General Medicine, Flinders Medical Centre, Adelaide, South

Australia, Australia

${ }^{2}$ College of Medicine and Public Health, Flinders University, Adelaide, South

Australia, Australia

Full list of author information is available at the end of the article
}

\section{$\int$ Bïomed Central}

(c) The Author(s). 2018 Open Access This article is distributed under the terms of the Creative Commons Attribution 4.0 International License (http://creativecommons.org/licenses/by/4.0/), which permits unrestricted use, distribution, and reproduction in any medium, provided you give appropriate credit to the original author(s) and the source, provide a link to the Creative Commons license, and indicate if changes were made. The Creative Commons Public Domain Dedication waiver (http://creativecommons.org/publicdomain/zero/1.0/) applies to the data made available in this article, unless otherwise stated. 


\section{Background}

Malnutrition is common in older hospitalized patients with prevalence rates as high as $30 \%$ in acute care settings in Australia [1]. Malnutrition is associated with adverse clinical outcomes for patients in terms of higher morbidity and mortality [2] and is costly for the hospitals [3]. The adverse effects associated with malnutrition on patient outcome and recovery results in increased health care use and costs [4]. Health-care costs are increased because malnourished patients stay longer in hospitals, suffer more infectious and non-infectious nosocomial complications, experience frequent hospital re-admissions and have higher utilization of health-care resources in the community [5-8]. Three recent meta-analyses [9-11] have indicated that nutrition intervention has economic benefits but have also suggested that there is a need for further high quality studies to confirm these findings in different age groups and in different health care settings. This is especially so as majority of these studies have been conducted in Europe and very few studies are available in the Australian health care settings.

A recent randomized controlled trial [12] conducted in a large tertiary hospital in Australia from 2014 to 2016, assessed efficacy of an early and extended nutrition intervention in older hospitalized patients. In this trial, an individualized nutrition intervention was started within $24 \mathrm{~h}$ of hospital admission and patients $\geq 60$ years age received monthly telehealth follow up for two months following discharge and this intervention was compared to usual care. The main objectives in this trial was to examine whether such an intervention could improve nutritional status and quality of care by reducing adverse clinical outcomes and optimizing use of existing resources.

This trial found a trend towards an improvement in nutritional status and quality of life and a significant reduction in length of hospital stay but there was no reduction in mortality or readmissions at 3 months follow up. Although the resources needed for the intervention were modest and the anticipated improvement in the nutrition status was small [12], no economic evaluation was conducted to examine whether the intervention was worth pursuing from an economic perspective. The objective of the present analysis was to conduct an economic evaluation that assessed whether the individualized nutrition intervention was value for money when considered from a healthcare sector (Australian Medicare) perspective. The results of the evaluation will help determine whether allocation of resources for improvement of nutritional status of older hospitalized patients is justifiable. Consequently, the primary outcome of this evaluation was expressed in terms of incremental costs per unit improvement in the PG-SGA (CEA) and the secondary outcome reported in terms of incremental costs per QALY gained (CUA).

\section{Methods \\ Study design}

The data for this health economic analysis were obtained from a recently conducted nutrition intervention study [12], which was designed as a randomized controlled trial.

\section{Target population}

The participants for this study included hospitalized patients aged $\geq 60$ years, who were confirmed as malnourished by a qualified dietitian using PG-SGA tool [13].

\section{Sample size}

The sample size was calculated based upon the change in the PG-SGA score from the baseline in the clinical trial [12] which provided data for this economic evaluation. The sample size in the clinical trial was based on the findings of a previous study [14], which has suggested that a shift of 3 (SD 4.1) in PG-SGA is clinically meaningful, assuming an affect size of 0.35 , alpha $=0.05$ and power of $80 \%$ the estimated sample size was 86 (43 in each group) was calculated to be sufficient.

\section{Setting and location}

This study included patients presenting to the Department of General Medicine, Flinders Medical Centre (FMC), Adelaide, South Australia. FMC is a tertiary level, teaching hospital with 520 beds capacity and the Department of General Medicine admits approximately 4500 patients per year. Health services at FMC are predominantly funded through the Australian Medicare Scheme (the primary funder of universal healthcare insurance in Australia). Patients were excluded if they were receiving palliative care, residing in rural areas, or were of indigenous origin or were non-English speaking. Rural, indigenous and non-English speaking subjects were excluded due to lack of funds to travel to rural areas for assessments and seek services of an Indigenous liaison officer/interpreter.

\section{Study perspective}

The direct costs of implementing nutritional intervention were determined from the Australian (Medicare) health care perspective. These included costs of hospitalizations, dietitian costs for post-discharge telephone calls, costs of providing nutrition supplements, post-discharge general practioner (GP) and specialist physician visits. Other costs were for any outpatient investigations and procedures, allied health care utilization and medicinal products over the period of 3-months of intervention. Indirect costs, such as those incurred by the patients due to loss of productivity were not included in this analysis. 


\section{Comparators}

The economic evaluation determined the relative costeffectiveness/cost-utility of the intervention when compared to the control.

\section{Intervention}

Nutrition intervention was initiated within 24 h of hospital admission and aimed to meet $100 \%$ of patients' energy and protein requirements for ideal body weight, calculated using commonly adopted predictive equations [15] along with an adequate intake of essential vitamins and minerals. Intervention patients received an individualized nutrition intervention by the dietitian, depending upon their underlying medical conditions, protein, energy, vitamin and mineral requirements and food preferences. Nutritional strategies employed by the dietitian included provision of oral nutrition supplements (ONS) $(1-2.2 \mathrm{kcal} / \mathrm{ml}$ and $0.05-0.12 \mathrm{~g}$ of protein $/ \mathrm{ml})$, mid-meal snacks and food fortification with consideration given to individual patients' food preferences and taste. The ONS utilized were Resource (Nestle Heath Science) (475 kcal, $19.7 \mathrm{~g}$ protein) and Sustagen (Nestle Heath Science) (248 kcal, 12.5 g protein), which in addition to protein provided a range of nutrients. Multivitamins were not separately prescribed but were left to the discretion of the treating clinicians. In addition, the patients and their care-providers received dietetic counseling, to augment their energy intake by using a range of strategies including recommendation of energy and nutrient dense food items, increasing the number of meals they ate, and consumption of energy, protein and nutrient-rich snacks. Patients who needed assistance with meals were flagged, so that a ward based staff member provided help during meals. The frequency of contact between patient and dietitian during the hospital stay varied depending upon individual patients' needs and the length of hospital stay. If the dietitian thought that the patient was unable to achieve their daily energy and nutrient requirements then they received almost daily input. Where patients were discharged to a nursing home then the dietitian contacted the nursing home manager and forwarded the recommended nutritional care plan to be followed. The hospital covered the cost of commercial oral nutritional supplements at the time of discharge for patients where $\geq 50 \%$ of the patient's daily energy requirements were determined to be required from supplements. All intervention patients were contacted by a monthly telephone call by the research dietitian for 2 months. During this interview, a structured format was used by the dietitian to collect information about patients' recent weight, compliance with the dietetic plan and any side effects with supplementation. In addition, patients received dietetic counseling with a focus to reinforce compliance with the intervention. Compliance with the dietetic plan was assessed by using a 24-h self-reported dietary recall. In this trial, the dietitian assessed the patients as compliant to the nutritional care plan if they were able to meet at least $75 \%$ of their energy and protein requirements.

\section{Control group}

Patients randomized to the control group followed usual care currently operative in Flinders Medical Centre. Currently all patients undergo nutrition screening by the use of Malnutrition Universal Screening Tool (MUST) and patients identified as high risk for malnutrition are referred to the dietitian. However, dietetic input occurs only if clinicians refer the patients and even if a dietitian sees them during hospital admission, they may not be followed after discharge. In this study, the control patients were flagged as malnourished and this was documented in the case notes for clinicians to make decisions regarding nutritional care. If control patients got referred for a dietetic advice, then they were offered the same nutritional care plan as the intervention group only for the period of their hospitalization but received no post discharge follow up care.

\section{Time horizon}

The costs between the two groups were compared over a period of three months from the time of randomization during hospital admission until the last follow-up.

\section{Discount rates}

Discounting of costs and effectiveness measures was not performed, because the time horizon of this study did not exceed 1 year [16].

\section{Choice of nutritional/health outcomes}

The primary nutritional outcome in this study, as was the case in the clinical study [12] and for the sake of maintaining consistency, was the unit improvement in the PG-SGA over the 3-month study period. The secondary outcome was QALYs gained over the same period and based on the responses to the EuroQoL $5 \mathrm{Di}$ mensions 5 Levels (EQ-5D-5 L) [17].

\section{PG-SGA}

The nutrition status of the participants was confirmed with PG-SGA by an experienced dietitian. The PG-SGA [18] generates a numerical score while also providing an overall global rating divided into three categories: well nourished (PG-SGA A), moderately malnourished or suspected of being malnourished (PG-SGA B) or severely malnourished (PG-SGA C). For each component of the PG-SGA, points (0-4) are awarded depending on the impact on nutritional status. Component scores are summed up to obtain total scores that range from 0 to 35 and scores $\geq 7$ indicating a critical need for nutritional 
intervention and symptom management in the older patients [19]. PG-SGA has been validated in various settings including older hospitalized patients and has a high sensitivity and specificity to diagnose malnutrition [19].

\section{HRQoL and QALYs}

QALYs gained were chosen as an outcome as they facilitate comparisons between interventions for disparate services and are recommended for use by decision makers including the Pharmaceutical Benefits Advisory Committee (PBAC) in Australia [20].

QALY estimates, calculated using the area-under-thecurve method [16], were based on responses to the EQ5D-5 L which were scored using UK value sets [17].

The EQ-5D-5 L is a self-reported questionnaire and measures a patient's health across five different domains: mobility, self-care, usual activities, pain/discomfort and anxiety/depression [21]. Using these responses, the EQ$5 \mathrm{D}-5 \mathrm{~L}$ is able to distinguish between 3125 states of health. A UK-specific algorithm developed using timetrade-off techniques was used to convert the EQ-5D-5 L health description into a valuation ranging from -0.281 to 1 [17]. Scores less than 0 represent health states that are worse than death [22]. The EQ-5D-5 L has been validated in different clinical populations including patients with multiple chronic illnesses, rehabilitation and orthopedic patients awaiting joint replacement surgery and has been found to have a stronger convergent validity coefficient (Spearman's coefficient 0.51-0.75) and a higher absolute informativity (Shannon's index) as compared to the EuroQol 5 Dimensions 3 Levels (EQ-5D-3 L) [23-25].

\section{Measurement of effectiveness}

No effectiveness data were obtained from secondary sources as our analysis relied upon data from our original trial [12].

\section{Estimating resources and cost}

Data on the volume and total costs of healthcare utilisation, measured from the health care perspective, were readily provided by Medicare Australia. Cost data were provided in the form of Medicare Benefits Schedule (MBS) data (number and costs of GP visits, specialist attendances, non-specialist attendance, diagnostic procedures and other medical services such as pathology and teleheath services); Pharmaceutical Benefits Schedule (PBS) data (quantity and costs of pharmaceuticals) and; centralised costing (Australian refined diagnosis related group (AR-DRG)) data [26] (number and costs of public hospital inpatient episodes). Patient consent was sought before obtaining MBS, PBS and AR-DRG data. Costs associated with the intervention itself (primarily dietitian staff costs for making follow-up telephone calls (30 $\mathrm{min}$ per month for two months i.e. two phone calls per patient for all patients) and costs of supplements for the entire study period for nearly half (36) of the patients) were estimated by combining staff time spent/ number of supplements provided and published information on wage rates obtained from published resources ( $\$ 37.16$ per hour for an accredited dietitian) and unit costs for supplements sourced from hospital accounts records ( $\$ 6$ per package per day). All costs are reported in Australian dollars at 2016/17 unit prices [16].

\section{Analytical methods \\ Descriptive statistics}

Continuous variables were expressed as mean (standard deviation (SD)) values or median (interquartile (IQR)) ranges and were compared using an appropriate parametric (Student $\mathrm{t}$ ) test or nonparametric (Mann-Whitney $\mathrm{U}$ ) test. Categorical variables were expressed as frequencies and percentages and were compared using Chi-square $\left(\mathrm{X}^{2}\right)$ statistics or Fishers exact test as appropriate. Length of hospital stay (LOS) was adjusted for in-hospital mortality.

\section{Economic evaluation}

Two types of economic evaluation (CEA and CUA) were used in this study. Their choice was informed by the types of outcomes measured in the main trial [12]. CEA is a type of economic evaluation whose outcomes are expressed in terms of natural units such as life expectancy or change in PG-SGA scores, while outcomes in CUA are reported in terms of QALYs [27]. Consequently, the primary outcome of this evaluation was expressed in terms of incremental costs per unit improvement in PG-SGA (CEA) and the secondary outcome reported in terms of incremental costs per QALYs gained (CUA). An incremental approach was used in order to determine, where appropriate, the incremental cost effectiveness ratios (ICERs) expressed as the incremental cost per unit improvement in the PG-SGA (primary outcome) and incremental costs per quality adjusted life year QALY gained (secondary outcome). The ICERs were calculated as incremental costs divided by incremental changes in outcomes. The economic evaluation was conducted using an intention-to-treat approach.

Within-trial economic evaluation with respect to the primary and secondary outcomes was undertaken allowing for bivariate uncertainty with bootstrapping of participant costs and outcomes to maintain the covariance structure. To account for uncertainty due to sampling variation in cost-effectiveness/cost-utility, nonparametric bootstrapping [28] were applied on participant level data to derive 5000 paired estimates of mean differences in costs and outcomes. These bootstrapped pairs were summarized within cost effectiveness planes (CEPs) [29]. The probability of the intervention being more cost effective, compared to 
the usual care arm at different willingness-to-pay thresholds, was depicted using Cost effectiveness acceptability curves (CEACs).

Due to the presence of missing data on costs and outcomes (Tables 1, 2 and 3), multiple imputation was used to account for missing values prior to conducting the base-case economic evaluation [30]. Imputed values were generated by use of an iterative Markov chain Monte Carlo method premised on multivariate normal regression. To appropriately characterize the uncertainty about the right value to impute, each missing value in the dataset was replaced with a set of 50 plausible values. Standard complete-case procedures were then applied to each of the 50 resultant multiply imputed datasets before combining the results using Rubin's rules [31]. The following variables were used to predict missing values in the imputation procedure: study arm, age, gender, cognitive status, length of stay, total number of comorbidities and malnutrition diagnosis. In both the base-case and sensitivity analyses, only adjusted outcomes (adjusted for baseline differences and correlation between repeated measurements) were used.
Sensitivity analyses were carried out to test the robustness of the base case results and they focused on evaluating the effect of missing cost and outcome data values on the economic evaluation results (i.e. comparing results based on complete cases and those estimated using multiple imputed values). All analyses were conducted in Microsoft Excel (2010) and Stata version 14.1.

\section{Results}

\section{Descriptive statistics}

A total of 1668 patients (Fig. 1) admitted to the Department of General Medicine were assessed for participation in this study, whereof 892 met the inclusion criteria. Of the 892,744 patients refused to participate due to various reasons (Fig. 1). One hundred and forty eight patients were therefore recruited and randomized to the control $(n=70)$ and intervention $(n=78)$ groups. The baseline clinical characteristics (Table 1) were similar between the two groups with regard to age, gender distribution, Charlson comorbidity index, number of medications and principal clinical diagnosis. There was no difference in severity of malnutrition at baseline as determined by PG-SGA

Table 1 Baseline characteristics of participants

\begin{tabular}{|c|c|c|c|}
\hline Characteristics & $\begin{array}{l}\text { Control } \\
(n=70)\end{array}$ & $\begin{array}{l}\text { Intervention } \\
(n=78)\end{array}$ & $P$ value \\
\hline Age, mean $(95 \% \mathrm{Cl})$, y & 81.6 (79.5 to 83.6$)$ & $82.0(80.0$ to 83.9$)$ & 0.76 \\
\hline \multicolumn{4}{|l|}{ Gender, n (\%) } \\
\hline Male & $23(32.9)$ & $31(39.7)$ & \\
\hline Female & $47(67.1)$ & $47(60.3)$ & 0.38 \\
\hline \multicolumn{4}{|l|}{ Residence before admission, $\mathrm{n}(\%)$} \\
\hline Home & $66(94.3)$ & $68(87.2)$ & 0.11 \\
\hline Nursing Home & $4(5.7)$ & 10 12.8) & \\
\hline \multicolumn{4}{|l|}{ Cognition, n (\%) } \\
\hline Normal & $67(95.7)$ & $74(94.9)$ & 0.56 \\
\hline Impaired & $3(4.3)$ & $4(5.1)$ & \\
\hline No of co-morbidities, mean ( $95 \% \mathrm{Cl})$ & $6.3(5.6$ to 6.9$)$ & $6.1(5.5$ to 6.6$)$ & 0.64 \\
\hline Charlson index, mean (95\% Cl) & 2.3 (1.9 to 2.8$)$ & 2.2 (1.8 to 2.7$)$ & 0.82 \\
\hline Medications at admission, mean $(95 \% \mathrm{Cl})$ & 10.1 (9.0 to 11.2$)$ & 8.8 (7.8 to 9.7$)$ & 0.07 \\
\hline \multicolumn{4}{|l|}{ Principal diagnosis at admission, n (\%) } \\
\hline Respiratory & $29(41.4)$ & $20(25.6)$ & 0.30 \\
\hline Cardiovascular & $8(11.4)$ & $14(18.0)$ & \\
\hline Falls & $10(14.3)$ & $13(16.7)$ & \\
\hline CNS & $3(4.3)$ & $6(7.7)$ & \\
\hline Miscellaneous & $20(28.6)$ & $25(32.1)$ & \\
\hline BMI, mean $(95 \% \mathrm{Cl}), \mathrm{kg} / \mathrm{m} 2$ & 21.8 (20.7 to 22.8$)$ & 20.6 (19.7 to 21.5$)$ & 0.09 \\
\hline PG-SGA score, mean (95\% Cl) & $13.3(12.2$ to 14.5$)$ & 12.1 (11.0 to 13.2$)$ & 0.11 \\
\hline EQ-5D-5 L index & 0.6746 (0.617 to 0.729$)$ & 0.6934 (0.638 to 0.746$)$ & 0.62 \\
\hline
\end{tabular}

Abbreviations: Cl Confidence Interval, CNS Central Nervous System, BMI Body Mass Index PG-SGA, Patient Generated Subjective Global Assessment, EQ-5D-5 L EuroQol 5 Dimensions 5 Levels 
Table 2 Mean costs per patient (AU \$)

\begin{tabular}{|c|c|c|c|c|c|}
\hline \multirow{2}{*}{$\begin{array}{l}\text { Costs }^{a} \\
\text { Base Case Analysis (imputed cases) }^{\mathrm{b}}\end{array}$} & \multicolumn{2}{|c|}{ Control } & \multicolumn{2}{|c|}{ Intervention } & \multirow[t]{2}{*}{ Difference (Bootstrapped 95\% Cl } \\
\hline & $n$ & Mean & $\mathrm{n}$ & Mean & \\
\hline \multicolumn{6}{|l|}{3 month MBS costs } \\
\hline GP Costs & 70 & $347(38)$ & 78 & $311(32)$ & $-37(-134,59)$ \\
\hline Specialist Attendance Costs & 70 & $20(5)$ & 78 & 12 & $-7(-19,4)$ \\
\hline Non-Specialist Attendance Costs & 70 & $251(43)$ & 78 & $243(36)$ & $-8(-122,100)$ \\
\hline Diagnostic Procedures costs & 70 & $200(40)$ & 78 & $197(31)$ & $-4(-111,94)$ \\
\hline Other Medical Service costs ${ }^{c}$ & 70 & 396 & 78 & $253(34)$ & $-143(-291,2)$ \\
\hline Total MBS costs & 70 & $1216(128)$ & 78 & $1008(97)$ & $-208(-529,149)$ \\
\hline \multicolumn{6}{|l|}{3 month PBS costs } \\
\hline Total drug costs & 70 & $838(186)$ & 78 & $601(57)$ & $-237(-703,47)$ \\
\hline \multicolumn{6}{|l|}{3 month Inpatient (DRG) costs } \\
\hline Total DRG costs & 70 & $13,882(1390)$ & 78 & $13,134(1439)$ & $-748(-4584,3310)$ \\
\hline \multicolumn{6}{|l|}{ Intervention costs } \\
\hline Total intervention costs & 70 & 0 & 78 & $286(30)$ & $286(225,352)$ \\
\hline Total Costs & 70 & $15,936(1397)$ & 78 & $15,029(1430)$ & $-907(-4854,2956)$ \\
\hline \multicolumn{6}{|l|}{ Sensitivity analysis (complete cases) ${ }^{d}$} \\
\hline \multicolumn{6}{|l|}{3 month MBS costs } \\
\hline GP Costs & 62 & $348(43)$ & 65 & 307 (39) & $-41(-151,92)$ \\
\hline Specialist Attendance Costs & 62 & 21 & 65 & 13 & $-9(-20,8)$ \\
\hline Non-Specialist Attendance Costs & 62 & $247(48)$ & 65 & $251(48)$ & $4(-108,142)$ \\
\hline Diagnostic Procedures costs & 62 & $200(42)$ & 65 & $211(39)$ & $10(-108,121)$ \\
\hline Other Medical Service costs ${ }^{c}$ & 62 & $389(73)$ & 65 & $248(47)$ & $-141(-334,5)$ \\
\hline Total MBS costs & 62 & $1205(143)$ & 65 & $1029(132)$ & $-176(-495,226)$ \\
\hline \multicolumn{6}{|l|}{3 month PBS costs } \\
\hline Total drug costs & 59 & $855(217)$ & 65 & $610(65)$ & $-245(-832,99)$ \\
\hline \multicolumn{6}{|l|}{3 month Inpatient (DRG) costs } \\
\hline Total DRG costs & 70 & $13,882(1390)$ & 78 & 13,134 (1439) & $-748(-3310,4584)$ \\
\hline \multicolumn{6}{|l|}{ Intervention costs } \\
\hline Total intervention costs & 70 & 0 & 78 & $286(30)$ & $286(225,352)$ \\
\hline Total Costs & 59 & $17,024(1595)$ & 60 & $12,078(917)$ & $-4947(-9030,-1451)$ \\
\hline
\end{tabular}

a MBS Medicare Benefits Schedule, PBS Pharmaceutical Benefits Schedule, DRG Australian Refined Diagnosis Related Groups (AR-DRGs) cost weights used to cost hospital admissions, GP General Practioner, Total costs = MBS costs + PBS costs + DRG costs + Intervention costs

${ }^{\mathrm{b}}$ Multiply imputed values. Multiple imputations carried out to account for up to 29 or $19 \%$ missing data on cost estimates

'Examples of other medical costs include pathology and telehealth services as well as allied-health care attendances

${ }^{\mathrm{d}}$ Analysis restricted to non-missing total cost estimates (119 or $81 \%$ )

score and HRQoL as determined by EQ-5D-5 L was similar between the two groups (Table 1). Nutritional intervention provided an additional mean $655(95 \%$ CI: 587.3 to 772.1$) \mathrm{kcal}$ of energy and 36.5 (95\% CI: 31.5 to 41.5$)$ grams of protein and $73 \%$ and $77.2 \%$ patients were compliant with the intervention at 1 month and 2 months post-discharge, respectively. Length of hospital stay (LOS) was significantly shorter in the intervention patients (9.9 (SD: 7.2)) vs. 6.9 (SD: 5.3), $\quad P<0.005)$ days, in control and intervention groups, respectively (Table 3 ).

\section{Incremental costs and outcomes}

\section{Base case analysis results}

Table 2 presents a breakdown of mean healthcare costs per participant over a 3 months follow-up period. In the base case, mean per participant total Australian Medicare costs were lower in the intervention group compared to the control arm (by $\$ 907$ per patient) but these differences were not statistically significant (95\% CI: $-\$ 2956$ to $\$ 4854)$. The main drivers of the higher costs in the control group were higher inpatient $(\$ 13,882$ versus $\$ 13,134)$ and drug (\$838 versus $\$ 601)$ costs. When 
Table 3 Outcomes of study

\begin{tabular}{|c|c|c|c|c|c|}
\hline \multirow[t]{2}{*}{ Outcomes $^{a}$} & \multirow[b]{2}{*}{$n$} & \multicolumn{2}{|l|}{ Control } & \multirow{2}{*}{$\begin{array}{l}\text { Intervention } \\
\text { Mean }\end{array}$} & \multirow[t]{2}{*}{ Difference (Bootstrapped 95\% Cl) } \\
\hline & & Mean & $n$ & & \\
\hline \multicolumn{6}{|l|}{ Base Case Analysis (imputed cases) } \\
\hline \multicolumn{6}{|l|}{ EQ-5D-5 $L$ and QALY gains } \\
\hline EQ-5D-5 L at baseline & 70 & $0.6746(0.0284)$ & 78 & $0.6934(0.0276)$ & $0.1088(-0.0489,0.0916)$ \\
\hline EQ-5D-5 L at 3 months & 70 & $0.5787(0.0407)$ & 78 & $0.6358(0.0349)$ & $0.0571(-0.0556,0.1560)$ \\
\hline Unadjusted QALYs & 70 & $0.1578(0.0064)$ & 78 & $0.1659(0.0067)$ & $0.0081(-0.0090,0.0265)$ \\
\hline Adjusted $^{\complement}$ QALYs & & & & & $0.005(-0.0079,0.0199)$ \\
\hline \multicolumn{6}{|l|}{ PG-SGA Scores } \\
\hline PG-SGA Scores at baseline & 70 & $13.3286(0.5817)$ & 78 & $12.1123(0.4951)$ & $-1.2163(-2.6163,0.1793)$ \\
\hline PG-SGA Scores at 3 months & 70 & $7.3770(0.4098)$ & 78 & $5.9136(0.4054)$ & $-1.4634(-2.4801,-0.1896)$ \\
\hline Unadjusted improvement in PG-SGA Scores ${ }^{d}$ & 70 & $5.9516(0.6594)$ & 78 & $6.1987(0.5547)$ & $0.2471(-1.4931,1.8661)$ \\
\hline Adjusted ${ }^{c}$ improvement in PG-SGA Scores ${ }^{d}$ & & & & & $1.3238(0.0240,2.3858)$ \\
\hline \multicolumn{6}{|l|}{ Inpatient stay } \\
\hline LOS in days & 69 & $9.9(7.2)$ & 71 & $6.9(5.3)$ & $3.0(0.9,5.1)$ \\
\hline \multicolumn{6}{|l|}{ Sensitivity analysis (complete cases) ${ }^{e}$} \\
\hline \multicolumn{6}{|l|}{ EQ-5D-5 L and QALY gains } \\
\hline EQ-5D-5 $L$ at baseline & 69 & $0.6736(0.0290)$ & 77 & $0.6926(0.0272)$ & $0.0189(-0.0537,0.1003)$ \\
\hline EQ-5D-5 L at 3 months & 60 & $0.5672(0.0487)$ & 69 & $0.6360(0.0407)$ & $0.0688(-0.0553,0.2043)$ \\
\hline Unadjusted QALYs & 59 & $(0.1553(0.0076)$ & 69 & $0.1658(0.0075)$ & $0.0105(-0.0096,0.0291)$ \\
\hline Adjusted $^{\complement}$ QALYs & & & & & $0.0060(-0.0086,0.0216)$ \\
\hline \multicolumn{6}{|l|}{ PG-SGA Scores } \\
\hline PG-SGA Scores at baseline & 69 & $13.3478(0.5848)$ & 74 & $12.0946(0.5240)$ & $-1.2532(-2.9491,0.2727)$ \\
\hline PG-SGA Scores at 3 months & 46 & $6.9783(0.6167)$ & 57 & $5.8070(0.5185)$ & $-1.712(-2.7446,0.3698)$ \\
\hline Unadjusted improvement in PG-SGA Scores ${ }^{d}$ & 46 & $6.1739(0.8876)$ & 57 & $5.8596(0.7081)$ & $-0.3143(-2.4223,1.8485)$ \\
\hline Adjusted $^{c}$ improvement in PG-SGA Scores ${ }^{d}$ & & & & & $0.9849(-0.5601,2.5912)$ \\
\hline
\end{tabular}

${ }^{a}$ EQ-5D-5 L EuroQoL 5 Dimensions 5 Levels, QALY Quality Adjusted Life Years, PG-SGA Patient Generated Subjective Global Assessment, LOS Length of Hospital Stay

${ }^{\mathrm{b}}$ Multiply imputed values. Multiple imputations carried out to account for up to $12 \%$ of the EQ-5D-5 L utility scores ( 2 or $1 \%$ of baseline and 19 or $1 \%$ of 3 -month EQ-5D-5 L scores)

'These scores have been adjusted for baseline differences

${ }^{d}$ These PG-SGA scores were reverse scored so that a positive score reflects an improvement in nutrition status

'Trial participants with complete information on baseline and 3-month outcomes

the adjusted outcomes in the base case were considered (Table 3), the intervention was more effective than the control with participants in this arm reporting unit improvements in the PG-SGA that were higher by 1.3238 units (95\% CI: 0.0240 to 2.3858 ) and QALYs that were higher by 0.0050 QALYs gained per patient $(95 \%$ CI: -0.0079 to 0.0199$)$. In line with best practice guidelines [32, 33], ICERs relating to both the primary and secondary outcomes are not presented, as the intervention was both cheaper and more effective regardless of outcome considered.

The CEPs in the base case analysis (Fig. 2) shows some uncertainly in the cost-effectiveness results but most of the bootstrapped paired estimates of mean differences in costs and outcomes appear in southeast and south-west quadrants.
The CEACs (Fig. 3) show that the probability of the intervention being cost-effective at willingness to pay values as low as $\$ 1000$ per unit improvement in PGSGA scores was above $98 \%$ while it was $78 \%$ at a willingness to pay of $\$ 50,000$ per QALY gained, the implicit cost-effectiveness threshold used in Australia [34].

\section{Sensitivity analysis results}

In the base case analysis, multiple imputation was used to deal with the missing data on costs (29 observations or 20\%), PG-SGA scores (45 observations or $30 \%$ ) and EQ-5D-5 L responses (19 observations or 13\%). In the sensitivity analysis, ignoring the missing data and using complete case analysis (Tables 2 and 3 ) did not have an effect on the incremental effectiveness. This is because the intervention was still more effective by 0.9849 units 


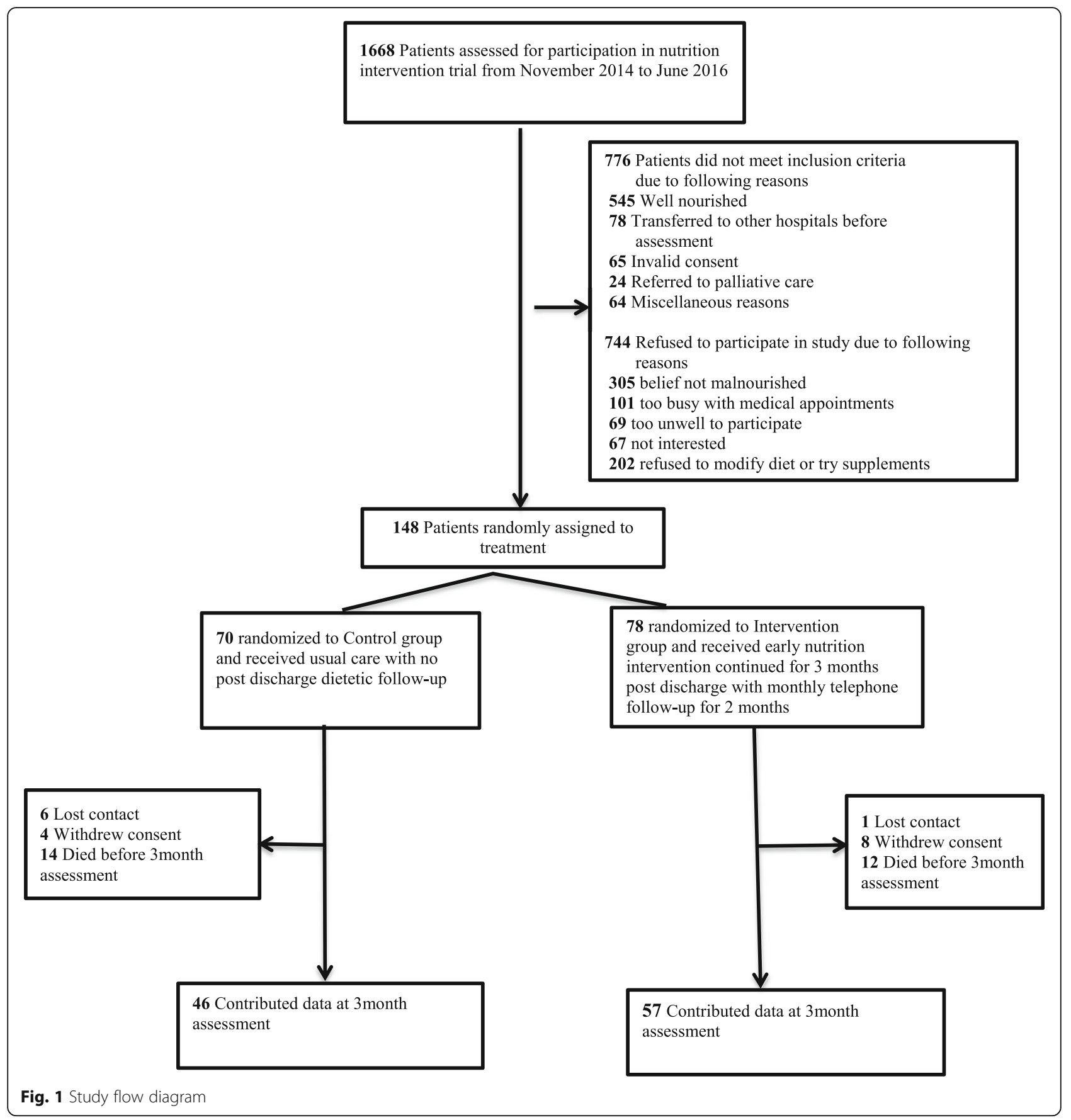

of improvement in the PG-SGA score (95\% CI: -0.5601 to 2.5912 ) and by 0.0060 QALYs gained per patient $(95 \%$ CI: -0.0086 to 0.0216 ), but was even more cheaper per patient (by $\$ 4947,95 \%$ CI: $\$ 1451$ to $\$ 9030$ ). These figures did not change the final interpretation because the intervention still outperformed the control.

\section{Discussion}

The findings of this study indicate that, in older general medical malnourished patients, the health care costs were lower while nutrition status and HRQoL was better among those in the individualized nutrition intervention arm compared to those in the group that received usual care with no post discharge dietetic follow-up. The differences in costs and HRQoL outcomes were however not statistically significant. In line with best practice guidelines [35, 36], therefore, our analysis focused on determining the likelihood of the intervention being costeffective as opposed to hypothesis testing relating to whether the cost and QALY differences were statistically 
a Improvement in Patient-Generated Subjective Global Assessment (PG-SGA) scores over 3 months

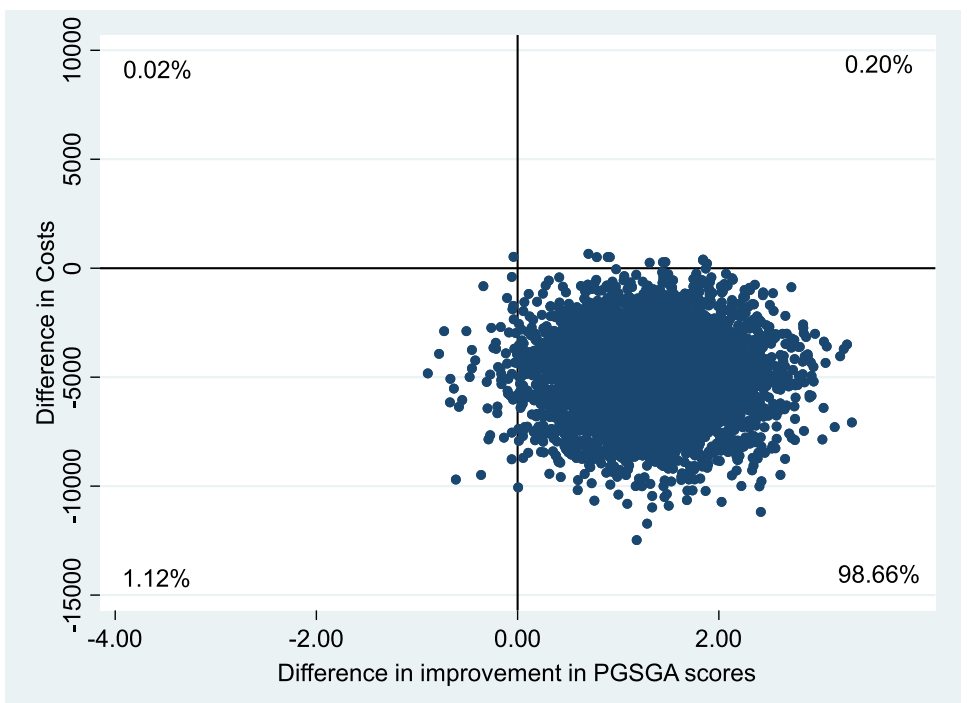

b Quality Adjusted Life Years (based on EuroQoL 5 dimensions 5 levels (EQ-5D-5L) responses) gained score over 3 months

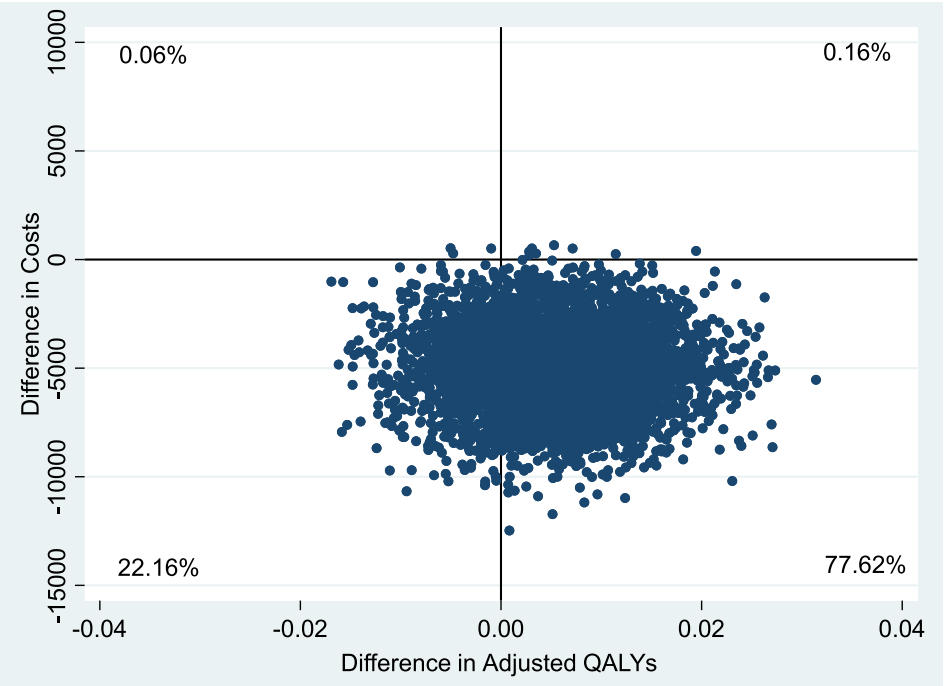

Fig. 2 Cost-Effectiveness Planes

significant. Our results show that probability of the intervention being cost-effective at willingness to pay values as low as $\$ 1000$ per unit improvement in PGSGA was $>98 \%$ while it was $78 \%$ at a willingness to pay $\$ 50,000$ per QALY gained. One of the strengths of this study is the use of PG-SGA for nutritional assessment, which has been demonstrated to have high sensitivity and specificity for the diagnosis of malnutrition and has been recommended as a predictive tool for clinical outcomes [14]. Yet, very few costing studies have utilized this stool for nutritional assessment.

At least two reasons may explain the statistically insignificant cost and HRQoL differences between the two trial arms. The first may be because the original trial [12] from which the data for this study were obtained was not powered to detect differences in costs and HRQoL, a result seen elsewhere [35, 36]. Another reason specific to HRQoL could be a short duration of nutrition intervention in our study. The impact of nutrition 
A Improvement in Patient Generated Subjective Global Assessment (PG-SGA) scores over 3 months

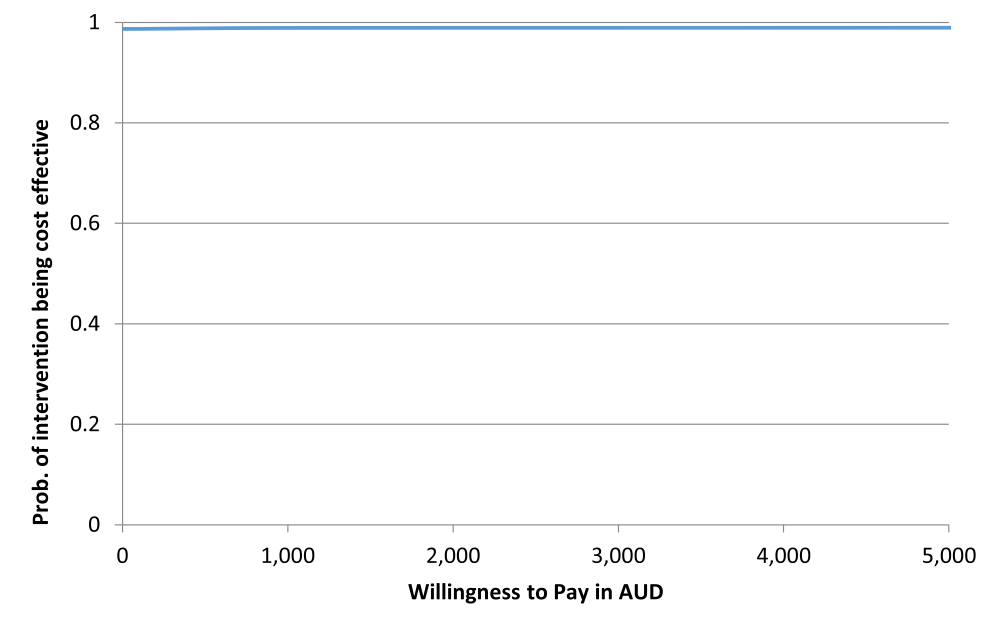

b Quality Adjusted Life Years (based on EuroQol 5 dimensions 5 levels (EQ-5D-5L) responses) gained score over 3 months

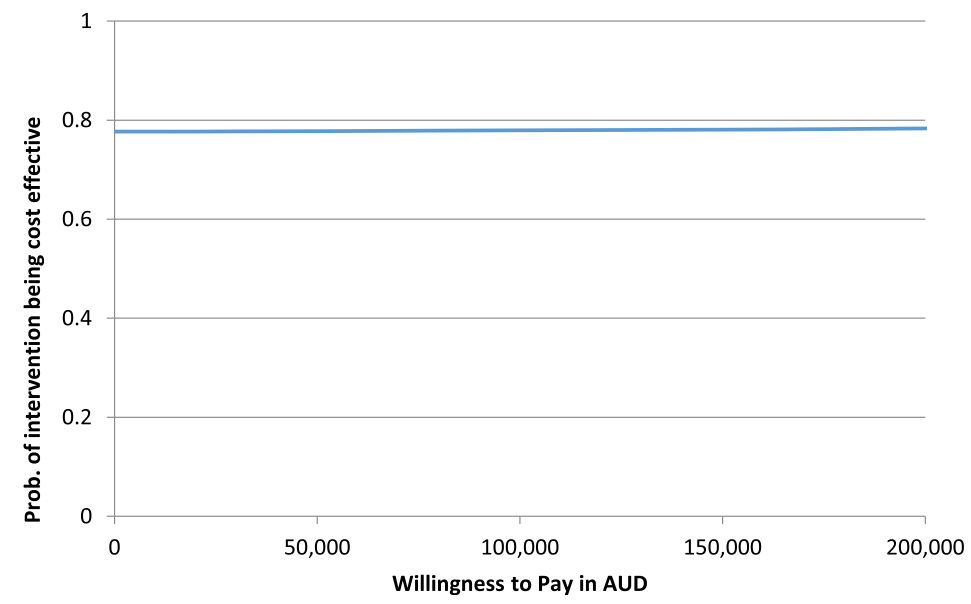

Fig. 3 Cost-Effectiveness Acceptability Curves

intervention on utilities is complex and may not be evident after a short period of intervention. After initiating nutrition intervention the temporal pattern that usually follows is - first improvement in nutrition parameters like weight then functional outcomes and lastly improvement in HRQoL [37]. Future nutrition intervention trials of sufficiently long duration may help verify this hypothesis.

The intervention was shown to have had lower mean Medicare costs than the control.

The cost drivers for the higher mean costs per patient in the control group were higher inpatient and drug costs. This could be related to the overall significantly longer length of hospital stay for the control patients with resultant higher utilization of health care resources. Studies have suggested that malnutrition contributes to the development of new complications such as delirium [38], predisposes to pressure ulcers [39] and increases risk of falls [40], all of which may contribute to the prolongation of the duration of hospitalization. Early nutrition intervention on the other hand may quickly improve the protein status and hence muscle function [41] as reflected by an increase in handgrip strength [42] and may lessen the risk of hospital acquired infections and may contribute to faster resolution of delirium [43]. It is possible that extension of this intervention following hospital discharge was associated with a sustained improvement in the nutrition status of intervention patients with a consequent reduction in the 'post-hospital syndrome' [44]. This may have led to a reduction in the utilization of 
primary health care resources (e.g. reduced GP visits) with consequent reduction in overall costs.

Our results are in line with a meta-analysis by Russell et al. [3] who found that use of ONS in surgical and elderly medical patients both in hospital and community settings can reduce LOS and complications with resultant net cost savings per patient. Our study is different from the studies used in the above meta-analysis in that we used a nutritional intervention tailored to individual patients needs rather than ONS alone, as studies have suggested poor compliance with ONS [45], especially in the older population. Similarly Gianotti et al. [46] found reduced treatment costs in patients who received enteral nutrition among patients undergoing major abdominal or cancer surgery and hypothesized that nutrition therapy helps improve splanchnic microperfusion with resultant lesser number of post-operative complications but in contrast to our study, this study included only surgical patients and limited nutrition intervention to the perioperative period. Norman et al. [47] in their study in malnourished patients aged $50.6 \pm 16.1$ years, with benign gastrointestinal disease found that 3-month nutritional supplementation with ONS increased HRQoL and was cost-effective from a German statutory health insurance perspective. Unlike our study, which included older patients with multiple comorbidities, however, this study was restricted to a relatively younger population of patients with benign gastrointestinal disease and nutrition intervention commenced only at the time of discharge. Our study results are also in line with the findings of three recent meta-analyses conducted in different patient groups [9-11], which suggest that the use of enteral medical nutrition in the management of disease related malnutrition (DRM) can be an efficient intervention from a health economic perspective and may lead to cost-savings.

Although malnutrition is common in older hospitalized patients, it is often poorly recognized by the clinicians with resultant fewer malnourished patients receiving treatment [48]. Economic evaluation offers a framework within which complex changes can be synthesized to aid in policy making. Our finding suggest that if similar intervention were to be delivered to all malnourished patients $\geq 60$ years of age in General Medical service of our hospital in 2015-16, a per-patient cost saving of $\$ 907$ will translate to a total savings of $\$ 1.86$ million and if applied to the State of South Australia total cost savings of $\$ 9.05$ million can be achieved. This study suggests that there is an opportunity to improve the health of malnourished older patients at a low marginal cost. Very few interventions have achieved health gains in this population at a lower cost [49]. In the current climate of economic constraints in healthcare, this study provides convincing evidence of the economic benefits of nutrition intervention.

\section{Limitations of study}

Although the use of a randomized controlled study provides robust evidence for assessing the utility of nutrition intervention, this study had limitations when assessing economic value. Our analysis did not consider several factors, which could bias the results by either underestimating or overestimating the cost-effectiveness of nutritional supplementation. While we included the direct medical costs, we did not consider broader or indirect costs such as those borne by patients and their families privately or by nursing homes and costs associated with loss of work due to periods of absence for patients or their carers. Additionally, our study duration is limited to 3 months and long-term impact of such a nutrition intervention is unknown. Our study did have missing data on some costs and outcomes, however principled and robust methods were used to deal with the missingness. Finally, the difference in QALY gains in this study can be considered to be small and therefore our result on the effectiveness should be interpreted with caution. The overall economic evaluation results nevertheless considered these QALY gains jointly with cost differences as is appropriate.

Due to differences in design and organization of health-care systems, our study results cannot be generalized to other settings and countries and further studies are needed to contribute to the evidence of cost utility of nutritional therapy.

\section{Implications}

Our study adds clinical and economic evidence of the benefits of initiating an early nutrition intervention with continuation in the community to improve health outcomes in older hospitalized malnourished population and justifies allocation of resources to improve the nutrition status of an elderly population.

\section{Conclusion}

For both primary (change in PG-SGA scores) and secondary outcomes (QALY gains), the results of our health economic analysis suggest that the use of early and extended nutritional intervention in older general medical patients is likely to be cost-effective in the Australian health care setting as the intervention was both cheaper and more effective than the comparator. This conclusion was supported further by results of the CEACs that showed that the intervention had a high likelihood of being the cost-effective option over a range of willingness to pay values.

\section{Abbreviations}

AR-DRG: Australian refined diagnosis related group; BMI: Body mass index; CEA: Cost effectiveness analysis; CEAC: Cost effectiveness acceptability curve; CEP: Cost effectiveness plane; CUA: Cost utility analysis; DRM: Disease related malnutrition; EQ-5D-5 L: European quality of life 5 dimension 5 level; 
HRQoL: Health related quality of life; LOS: Length of hospital stay; MBS: Medicare benefits schedule; MUST: Malnutrition universal screening tool; ONS: Oral nutritional supplements; PBAC: Pharmaceutical benefit advisory committee; PBS: Pharmaceutical benefits schedule; PG-SGA: Patient generated subjective global assessment; QALY: Quality adjusted life year

\section{Funding}

This study was not supported by any funding.

\section{Availability of data and materials}

The datasets used and analysed during the current study are available from the corresponding author on reasonable request only if permission can be granted by Medicare Australia.

\section{Authors' contributions}

All authors contributed substantially to this manuscript. YS, MM, CT and BK designed this study. Data acquisition was done by YS, RS, PH and CH. Statistical analysis was carried by $\mathrm{YS}, \mathrm{PH}$ and $\mathrm{CH}$. Economic analysis was done by BK. Data interpretation was done by $\mathrm{YS}, \mathrm{BK}, \mathrm{MM}, \mathrm{CT}, \mathrm{RS}, \mathrm{PH}$ and $\mathrm{CH}$. YS and BK drafted the manuscript. MM and $\mathrm{CT}$ revised it critically for intellectual content. All authors read and approved the final manuscript.

\section{Ethics approval and consent to participate}

This study was approved by Southern Adelaide Human Research Ethics (SA HREC) committee approval number 273.1. This trial was prospectively registered with Australia and New Zealand clinical trial registry No ACTRN12614000833662 on 6 August 2014. Written informed consent was taken from all the participants.

\section{Consent for publication}

Not applicable.

\section{Competing interests}

The authors declare that they have no competing interests.

\section{Publisher's Note}

Springer Nature remains neutral with regard to jurisdictional claims in published maps and institutional affiliations.

\section{Author details \\ 'Department of General Medicine, Flinders Medical Centre, Adelaide, South Australia, Australia. ${ }^{2}$ College of Medicine and Public Health, Flinders University, Adelaide, South Australia, Australia. ${ }^{3}$ Discipline of Medicine, University of Adelaide, Adelaide, South Australia, Australia. ${ }^{4}$ Department of Nutrition \& Dietetics, Flinders University, Adelaide, South Australia, Australia. ${ }^{5}$ Faculty of Health Sciences, Flinders University, Adelaide, South Australia, Australia. ${ }^{6}$ Department of Clinical Epidemiology, Flinders Medical Centre, Adelaide, South Australia, Australia. ${ }^{7}$ Health Economics Unit, Flinders University, Adelaide, South Australia, Australia.}

\section{Received: 19 July 2017 Accepted: 29 January 2018}

Published online: 05 February 2018

\section{References}

1. Charlton K, Nichols C, Bowden S, Milosavljevic M, Lambert K, Barone L, et al. Poor nutritional status of older subacute patients predicts clinical outcomes and mortality at 18 months of follow-up. Eur J Clin Nutr. 2012;66:1224-8.

2. Sharma Y, Thompson C, Shahi R, Hakendorf P, Miller M. Malnutrition in acutely unwell hospitalized elderly - "The skeletons are still rattling in the hospital closet". J Nutr 2017; doi:https://doi.org/10.1007/s12603-017-0903-61-6.

3. Russell CA. The impact of malnutrition on healthcare costs and economic considerations for the use of oral nutritional supplements. Clin Nutr. 2007;2:25-32.

4. Kruizenga HM, Van Tulder MW, Seidell JC, Thijs A, Ader HJ, Van Bokhorst-de van der Schueren MA. Effectiveness and cost-effectiveness of early screening and treatment of malnourished patients. Am J Clin Nutr. 2005;82:1082-9.

5. Agarwal E, Ferguson M, Banks M, Batterham M, Bauer J, Capra S, et al. Nutrition care practices in hospital wards: results from the nutrition care day survey 2010. Clin Nutr. 2012;31:995-1001.

6. Mudge AM, Kasper K, Clair A, Redfern H, Bell JJ, Barras MA, et al. Recurrent readmissions in medical patients: a prospective study. J Hosp Med. 2011;6:61-7.

7. Ljungqvist O, van Gossum A, Sanz ML, de Man F. The European fight against malnutrition. Clin Nutr. 2010;29:149-50.
8. Freijer K, Tan SS, Koopmanschap MA, Meijers JM, Halfens RJ, Nuijten MJ. The economic costs of disease related malnutrition. Clin Nutr. 2013;32:136-41.

9. Milte RK, Ratcliffe J, Miller MD, Crotty M. Economic evaluation for protein and energy supplementation in adults: opportunities to strengthen the evidence. Eur J Clin Nutr. 2013;67:1243-50.

10. Freijer K, Bours MJ, Nuijten MJ, Poley MJ, Meijers JM, Halfens RJ, et al. The economic value of enteral medical nutrition in the management of diseaserelated malnutrition: a systematic review. J Am Med Dir Assoc. 2014;15:17-29.

11. Elia M, Normand C, Norman K, Laviano A. A systematic review of the cost and cost effectiveness of using standard oral nutritional supplements in the hospital setting. Clin Nutr. 2016;35:370-80.

12. Sharma $Y$, Thompson C, Kaambwa B, Shahi R, Hakendorf P, Miller M. Investigation of the benefits of early malnutrition screening with telehealth follow up in elderly acute medical admissions. QJM 2017; doi:https://doi. org/10.1093/ajmed/hcx095.

13. Sharma Y, Thompson C, Kaambwa B, Shahi R, Miller M. Validity of the malnutrition universal screening tool (MUST) in Australian hospitalized acutely unwell elderly patients. Asia Pac J Clin Nutr. 2017;26:994-1000.

14. Isenring E, Bauer J, Capra S. The scored patient-generated subjective global assessment (PG-SGA) and its association with quality of life in ambulatory patients receiving radiotherapy. Eur J Clin Nutr. 2003;57(2):305-9.

15. Parker EA, Feinberg TM, Wappel S, Verceles AC. Considerations when using predictive equations to estimate energy needs among older, hospitalized patients: a narrative review. Curr Nutr Rep. 2017;6:102-10.

16. Drummond MF, Sculpher M, O'Brien B, Stoddart GW. Methods for the economic Evalution of health care Programmes. 3rd ed. Oxford: Oxford University Press; 2005.

17. Devlin NS, Shah KK.; Feng Y.; Mulhern B; van Hout B. Valuing health-related quality of life: an EQ-5D-5L value set for England. Health Econ 2017; doi: https://doi.org/10.1002/hec.3564.

18. Desbrow B, Bauer J, Blum C, Kandasamy A, McDonald A, Montgomery K. Assessment of nutritional status in hemodialysis patients using patientgenerated subjective global assessment. J Renal Nutr. 2005;15:211-6.

19. Marshall S, Young A, Bauer J, Isenring E. Malnutrition in geriatric rehabilitation: prevalence, patient outcomes, and criterion validity of the scored patient-generated subjective global assessment and the mini nutritional assessment. J Acad Nutr Diet. 2016;116:785-94.

20. Henry DA, Hill SR, Harris A. Drug prices and value for money: the Australian pharmaceutical benefits scheme. JAMA. 2005;294:2630-2.

21. Cheung K, Oemar M, Oppe M, Rabin R. EQ-5D user guide: basic information on how to use EQ-5D. Rotterdam: EuroQol Group; 2009.

22. Kind $\mathrm{P}$. The EuroQol instrument: an index of health-related qualilty of life. In: Spilker B, editor. Quality of life and Pharmacoeconomics in clinical trials. Philadelphia: Lippincott-Raven Publishers; 1996. p. 191-201.

23. Janssen MF, Pickard AS, Golicki D, Gudex C, Niewada M, Scalone L, et al. Measurement properties of the EQ-5D-5L compared to the EQ-5D-3L across eight patient groups: a multi-country study. Qual Life Res. 2013; 22:1717-27.

24. Conner-Spady BL, Marshall DA, Bohm E, Dunbar MJ, Loucks L, Al Khudairy A et al. Reliability and validity of the EQ-5D-5L compared to the EQ-5D-3L in patients with osteoarthritis referred for hip and knee replacement. Qual Life Res. 2015;24:1775-84.

25. Golicki D, Niewada M, Buczek J, Karlinska A, Kobayashi A, Janssen MF, et al. Validity of EQ-5D-5L in stroke. Qual Life Res. 2015;24:845-50.

26. Green JP, McNamee JP, Kobel C, Seraji MHR, Lawrence SJ. Planning for subacute care: predicting demand using acute activity data. Aus Health Rev. 2016:40:686-90.

27. Palmer S, Byford S, Raftery J. Economics notes: types of economic evaluation. BMJ. 1999;318:1349

28. Barber JA, Thompson SG. Analysis and interpretation of cost data in randomised controlled trials: review of published studies. BMJ. 1998;317:1195-200.

29. Black WC. The CE plane: a graphic representation of cost-effectiveness. Med Decis Mak. 1990;10:212-4.

30. Schafer UL. Analysis of incomplete multivariate data. London: Chapman \& Hall; 1997.

31. Rubin DB. Multiple imputation for nonresponse in surveys. New York: John Wiley \& Sons, Inc; 1987.

32. Husereau D, Drummond M, Petrou S, Carswell C, Moher D, Greenberg D, et al. Consolidated health economic evaluation reporting standards (CHEERS)explanation and elaboration: a report of the ISPOR health economic evaluation publication guidelines good reporting practices task force. Value Health. 2013;16:231-50. 
33. Joensuu JT, Huoponen S, Aaltonen KJ, Konttinen YT, Nordstrom D, Blom M. The cost-effectiveness of biologics for the treatment of rheumatoid arthritis: a systematic review. PLoS One. 2015;10:e0119683.

34. Harris AH, Hill SR, Chin G, Li JJ, Walkom E. The role of value for money in public insurance coverage decisions for drugs in Australia: a retrospective analysis 1994-2004. Med Decis Mak. 2008;28:713-22.

35. O'Sullivan AK, Thompson D, Drummond MF. Collection of health-economic data alongside clinical trials: is there a future for piggyback evaluations? Value Health. 2005;8:67-79.

36. Petrou S, Gray A. Economic evaluation alongside randomised controlled trials: design, conduct, analysis, and reporting. BMJ. 2011;342:d1548.

37. Neelemaat F, Bosmans JE, Thijs A, Seidell JC. Oral nutritional support in malnourished elderly decreases functional limitations with no extra costs. Clin Nutr. 2012;31:183-90.

38. Mazzola P, Ward L, Zazzetta S, Broggini V, Anzuini A, Valcarcel B, et al. Association between preoperative malnutrition and postoperative delirium after hip fracture surgery in older adults. J Am Geriatr Soc. 2017;65:1222-8.

39. Banks M, Bauer J, Graves N, Ash S. Malnutrition and pressure ulcer risk in adults in Australian health care facilities. Nutrition. 2010;26:896-901.

40. Misu S, Asai T, Doi T, Sawa R, Ueda Y, Saito T, et al. Association between gait abnormality and malnutrition in a community-dwelling elderly population. Geriatrics Gerontol Int 2016; https://doi.org/10.1111/ggi.12839.

41. Espaulella J, Guyer H, Diaz-Escriu F, Mellado-Navas JA, Castells M, Pladevall M. Nutritional supplementation of elderly hip fracture patients. A randomized, double-blind, placebo-controlled trial. Age Ageing. 2000;29:425-31.

42. Duncan DG, Beck SJ, Hood K, Johansen A. Using dietetic assistants to improve the outcome of hip fracture: a randomised controlled trial of nutritional support in an acute trauma ward. Age Ageing. 2006;35:148-53.

43. Rypkema G, Adang E, Dicke H, Naber T, de Swart B, Disselhorst L, et al. Costeffectiveness of an interdisciplinary intervention in geriatric inpatients to prevent malnutrition. J Nutr. 2004:8:122-7.

44. Krumholz HM. Post-hospital syndrome-an acquired, transient condition of generalized risk. N Engl J Med. 2013;368:100-2.

45. Bruce D, Laurance I, McGuiness M, Ridley M, Goldswain P. Nutritional supplements after hip fracture: poor compliance limits effectiveness. Clin Nutr. 2003;22:497-500.

46. Gianotti L, Braga M, Frei A, Greiner R, Di Carlo V. Health care resources consumed to treat postoperative infections: cost saving by perioperative immunonutrition. Shock. 2000;14:325-30.

47. Norman K, Pirlich M, Smoliner C, Kilbert A, Schulzke JD, Ockenga J, et al. Cost-effectiveness of a 3-month intervention with oral nutritional supplements in disease-related malnutrition: a randomised controlled pilot study. Eur J Clin Nutr. 2011;65:735-42.

48. Allard JP, Keller H, Jeejeebhoy KN, Laporte M, Duerksen DR, Gramlich L, et al. Malnutrition at hospital admission-contributors and effect on length of stay: a prospective cohort study from the Canadian malnutrition task force. JPEN J Parenter Enteral Nutr. 2016;40:487-97.

49. Zhong Y, Cohen JT, Goates S, Luo M, Nelson J, Neumann PJ. The CostEffectiveness of Oral Nutrition Supplementation for Malnourished Older Hospital Patients. Appl Health Econ Health Policy. 2016; doi:https://doi.org/ 10.1007/s40258-016-0269-7.

\section{Submit your next manuscript to BioMed Central and we will help you at every step:}

- We accept pre-submission inquiries

- Our selector tool helps you to find the most relevant journal

- We provide round the clock customer support

- Convenient online submission

- Thorough peer review

- Inclusion in PubMed and all major indexing services

- Maximum visibility for your research

Submit your manuscript at www.biomedcentral.com/submit

) Biomed Central 